\title{
Influence of an Inhomogeneous Ground on the Propagation of VLF Radio Waves in the Earth-Ionosphere Waveguide
}

\author{
James R. Wait \\ National Bureau of Standards, Boulder, Colo.
}

(Received February 11, 1965)

\begin{abstract}
Propagation of radio waves in the earth-ionosphere waveguide is considered for the case where the lower boundary is an inhomogeneous smooth surface. An integral equation for the problem is formulated in a direct fashion by utilizing the compensation theorem. After some simplifications, several special cases are considered explicitly. For example, in the case of a two-section path consisting of a long stretch of sea and a short section of land, a relatively simple working formula is obtained. The result shows that the modal excitation factors at VLF for an all sea path are significantly reduced when the foreground is poorly conducting. Another special case considered is when the propagation path is all sea except for a short intermediate land section. In this case, it is found that energy from loworder modes will be transferred to high-order modes with a subsequent reduction of field strength.
\end{abstract}

\section{Introduction}

A great deal of attention has been given to the prob. lem of predicting groundwave fields for mixed land/sea paths. Furthermore, extensive calculations have been made which show the interdependence of the various parameters. Since a recent review of progress in this area is now available [Wait, 1964], it is not necessary to discuss this particular topic here. However, it is rather surprising that, in propagation via ionospheric reflections, little attention has been given to the influence of an inhomogeneous earth. It is the purpose of this paper to consider this problem with special reference to $V L F$ radio propagation.

\section{Formulation}

The mutual impedance $z_{m}$ between two vertical antennas at A and B (separated by a great circle distance $d$ ) located on a spherical earth of radius $a$ is considered. In order to account for the presence of the ionosphere, an equivalent reflecting layer is located at height $h$. For present purposes, we assume this layer is characterized by a surface impedance $Z_{i}$ which does not vary along the path. If over the earth's surface between the terminals $A$ and $B$, the surface impedance is $Z$ everywhere, the mutual impedance may be expressed as a sum of modes as follows

$$
z_{m}=z_{0}\left[\frac{d / a}{\sin (d / a)}\right]^{1 / 2} W(Z, d)
$$

where $z_{0}$ is the mutual impedance between the dipoles $\mathrm{A}$ and $\mathrm{B}$ if they were located on a perfectly conducting flat ground plane and separated by a distance $d$. In the above expression, $W(Z, d)$ is an attenuation function defined by [Wait, 1962]:

$$
W(Z, d)=\frac{(d / \lambda)^{1 / 2}}{(h / \lambda)} e^{-i \pi / 4} \sum_{n} \exp \left(i k d \frac{C_{n}^{2}}{2}\right) \Lambda_{n},
$$

where $C_{n}$ is the cosine of a complex angle, and $\Lambda_{n}$ is the excitation factor for modes of order $n$. The $C_{n}$ 's are solutions of a modal equation which involves the following dimensionless parameters:

$$
\begin{aligned}
\left(-t_{n}\right)^{1 / 2} & =C_{n}(k a / 2)^{1 / 3} \\
y_{0} & =k h(k a / 2)^{-1 / 3} \\
q & =-i\left(Z / \eta_{0}\right)(k a / 2)^{1 / 3} \\
q_{i} & =-i\left(Z_{i} / \eta_{0}\right)(k a / 2)^{1 / 3},
\end{aligned}
$$

where $k=2 \pi /$ wavelength, and $\eta_{0}=120 \pi$ ohms. After making a number of simplifying assumptions, the modal equation mentioned above may be written in the form [Wait, 1962]

$\left[\frac{w_{2}^{\prime}\left(t_{n}\right)-q w_{2}\left(t_{n}\right)}{w_{1}^{\prime}\left(t_{n}\right)-q w_{1}\left(t_{n}\right)}\right]\left[\frac{w_{1}^{\prime}\left(t_{n}-y_{0}\right)+q_{i} w_{1}\left(t_{n}-y_{0}\right)}{w_{2}^{\prime}\left(t_{n}-y_{0}\right)+q_{i} w_{2}\left(t_{n}-y_{0}\right)}\right]=e^{-\mathrm{i} 2 \pi n,}$,

where $w_{1}(t)$ and $w_{2}(t)$ are Airy functions while the primes indicate derivatives with respect to the arguments. An alternative form of (2) is

$$
W(q, \chi)=\frac{2(\pi \chi)^{1 / 2}}{y_{0}} e^{-i \pi / 4} \sum_{n} \exp \left(-i \chi t_{n}\right) \Lambda_{n}
$$


where

$$
\chi=(d / a)(k a / 2)^{1 / 3} .
$$

Numerical values of $t_{n}$ and the excitation factor $\Lambda_{n}$ are now available for a wide range of parameters [Wait and Spies, 1964]. Thus, for the purposes of this communication, the solution of the waveguide problem with constant wall impedances $Z_{i}$ and $Z$ is taken to be known. What is of interest here is the extension to the same waveguide when the surface impedance of the lower boundary is a function of position along the path connecting $\mathrm{A}$ and $\mathrm{B}$. For example, as indicated in figure 1 , it is now assumed that, over some area $S$ on the earth's surface, the surface impedance is $Z^{\prime}$ which may be different from $Z$. The method of approach is very similar to that used by the author [Wait, 1964] in studying mixed-path groundwave propagation. As in that case, the problem may be formulated in terms of the compensation theorem in the form derived by Monteath [1951]. For example, the mutual impedance $z_{m}^{\prime}$ between dipoles $\mathrm{A}$ and $\mathrm{B}$ over an inhomogeneous ground of variable surface impedance $Z^{\prime}$ is given by

$$
z_{m}^{\prime}=z_{m}+\frac{1}{I_{0}^{2}} \iint_{S}\left(Z^{\prime}-Z\right) \mathbf{H}_{a t} \cdot \mathbf{H}_{b t}^{\prime} d S
$$

where $z_{m}$ is the mutual impedance if the surface of the earth were homogeneous with surface impedance $Z$ everywhere. The tangential magnetic field of dipole A over the homogeneous earth is $\mathbf{H}_{a t}$ while the tangential magnetic field over the inhomogeneous earth is $\mathbf{H}_{b t}^{\prime}$. The currents in the dipoles are both taken equal to $I_{0}$ for convenience. The surface of integration $S$ extends over the region of the earth which is characterized by a surface where $Z^{\prime}$ differs from $Z$ as indicated in figure 1 .

The formal equivalence of (5) with the formula of mixed-path groundwave theory is a result of the impedance boundary condition on the upper wall of the waveguide. In other words, the surface impedance $Z_{i}$ is assumed to be the same for both the homogeneous and for the inhomogeneous earth cases. If this were not permissible, there would be an additional surface integral over the upper boundary of the waveguide.

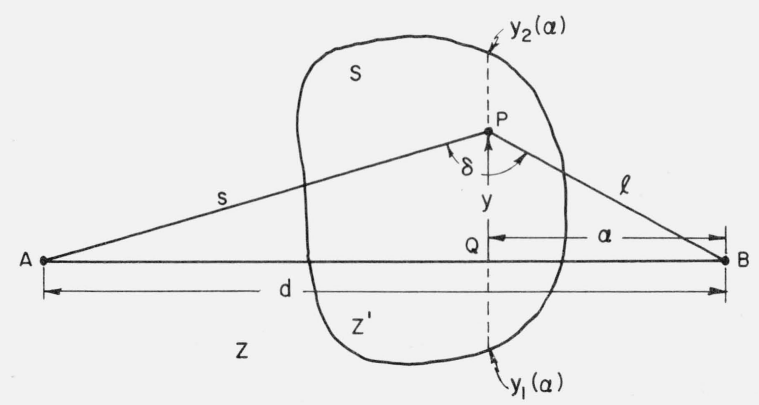

Figure 1. Plan view of the inhomogeneous region on the earth's surface.
To simplify the present problem, the mutual impedance $z_{m}^{\prime}$ is defined in terms of an attenuation $W^{\prime}\left(Z, Z^{\prime}, d\right)$ such that

$$
z_{m}^{\prime}=z_{0}\left[\frac{d / a}{\sin (d / a)}\right]^{1 / 2} W^{\prime}\left(Z, Z^{\prime}, d\right)
$$

in analogy to (1) for the homogeneous earth. The next step is to express the tangential magnetic field vectors, at the point of integration $P$ in terms of attenuation functions. Thus

$$
\mathbf{H}_{a t}=\frac{i k I_{0} h_{a}}{2 \pi s} e^{-i k s}\left(1+\frac{1}{i k s}\right)\left[\frac{s / a}{\sin (s / a)}\right]^{1 / 2} W(Z, s)\left(\mathbf{i}_{n} \times \mathbf{i}_{s}\right)
$$

and

$$
\begin{aligned}
\mathbf{H}_{b t}^{\prime}=\frac{i k I_{0} h_{b}}{2 \pi l} e^{-i k l}\left(1+\frac{1}{i k l}\right)\left[\frac{l / a}{\sin (l / a)}\right]^{1 / 2} \\
W^{\prime}\left(Z, Z^{\prime}, l\right)\left(\mathbf{i}_{n} \times \mathbf{i}_{l}\right),
\end{aligned}
$$

where $s$ and $l$ are great circle distances from A and B to $\mathrm{P}$ and $\mathbf{i}_{s}$ and $\mathbf{i}_{l}$ are unit vectors in the directions of increasing $s$ and $l$, respectively. In the above, $h_{a}$ and $h_{b}$ are the effective heights of the dipoles $\mathrm{A}$ and $\mathrm{B}$ while $\mathbf{i}_{n}$ is a unit vector normal to and into the surface of the spherical earth.

Equation (5), when combined with (6), (7), and (8), leads to the equation

$$
\begin{aligned}
& W^{\prime}\left(Z, Z^{\prime}, d\right)=W(Z, d)+\frac{i k d}{2 \pi T \eta_{0}} \iint_{S} G(s, l) \frac{e^{-i k(s+l-d)}}{s l} \\
& \times\left(Z^{\prime}-Z\right) W(Z, s) W^{\prime}\left(Z, Z^{\prime}, l\right) \cos \delta d S,
\end{aligned}
$$

where $\delta$ is the angle subtended by $\mathbf{i}_{s}$ and $\mathbf{i}_{l}$ and where

$$
\begin{aligned}
G(s, l) & =\left[\frac{s / a}{\sin (s / a)}\right]^{1 / 2}\left[\frac{l / a}{\sin (l / a)}\right]^{1 / 2}\left(1+\frac{1}{i k s}\right)\left(1+\frac{1}{i k l}\right) \\
T & =\left[\frac{d / a}{\sin (d / a)}\right]^{1 / 2}\left(1+\frac{1}{i k d}-\frac{1}{k^{2} d^{2}}\right)
\end{aligned}
$$

This is a two dimensional integral equation for the unknown function $W^{\prime}\left(Z, Z^{\prime}, d\right)$. To solve such an equation directly appears to be hopeless. Therefore, some simplifications are made at this stage in order to achieve tractability.

\section{Approximate Form of the Integral Equation}

We note that the function $\exp [-i k(s+l-d]$ is rapidly varying compared with other factors in the integrand. Therefore, one may expect that the principal contribution to the integrand will occur when $s+l \simeq d$, provided that the surface impedance contrast $Z^{\prime}-Z$ does not change rapidly in a direction transverse to the path. Therefore, in the other factors 
in the integrand, $l$ may be replaced by $\alpha$, and $s$ may be replaced by $d-\alpha$, where $\alpha$ is the great circle distance from $B$ to the point $Q$ on the great circle between $\mathrm{A}$ and $\mathrm{B}$. (The arc QP is perpendicular to AB.) Furthermore, over most of the range of integration, $(1 / k s)$ and $(1 / k l)$ may be neglected compared with unity and similarly $\cos \delta$ may be replaced by -1 . The latter approximations are obviously violated when the terminals are near discontinuities of the surface impedance $Z^{\prime}$.

The reduced form of the integral equation is

$W^{\prime}\left(Z, Z^{\prime}, d\right)-W(Z, d)=-\frac{i k d}{2 \pi \eta_{0}}$

$$
\iint_{S} f(\alpha) \frac{e^{-i k(s+l-d)}}{\alpha(d-\alpha)}\left[Z^{\prime}(\alpha)-Z\right]
$$

$$
\times W(Z, d-\alpha) W^{\prime}\left(Z, Z^{\prime}, \alpha\right) d S,
$$

where

$$
f(\alpha)=\left[\frac{\sin (d / a)}{(d / a)}\right]^{1 / 2}\left[\frac{\sin (\alpha / a)}{(\alpha / a)}\right]^{-1 / 2}\left[\frac{\sin [(d-\alpha) / a]}{[(d-\alpha) / a]}\right]^{-1 / 2}
$$

where all quantities, except the exponential factor, vary with $\alpha$ only. The exponent $s+l-d$ is now expressed in terms of the angle coordinates $\Omega$ and $\beta$ with reference to figure 2 ; these are defined by

$$
\Omega=\alpha / a=\mathrm{QB} / a \text { and } \beta=\mathrm{QP} / a
$$

From spherical trigonometry

$$
\cos (\mathrm{BOP})=\cos \Omega \cos \beta
$$

and

$$
\cos (\mathrm{AOP})=\cos (\theta-\Omega) \cos \beta .
$$

Thus,

$$
\mathrm{BOP}=\frac{l}{a}=\Omega+\left[\frac{\cot \Omega}{2}\right] \beta^{2}+\text { terms in } \beta^{4}, \beta^{6}, \ldots .
$$

and

$$
\begin{aligned}
\mathrm{AOP}=\frac{s}{a}=\theta-\Omega+\left[\frac{\cot (\theta-\Omega)}{2}\right] & \beta^{2} \\
& \quad+\text { terms in } \beta^{4}, \beta^{6}, \ldots .
\end{aligned}
$$

Therefore, to a first order in $\beta^{2}$,

$$
s+l-d \cong \frac{a}{2}[\cot \Omega+\cot (\theta-\Omega)] \beta^{2} .
$$

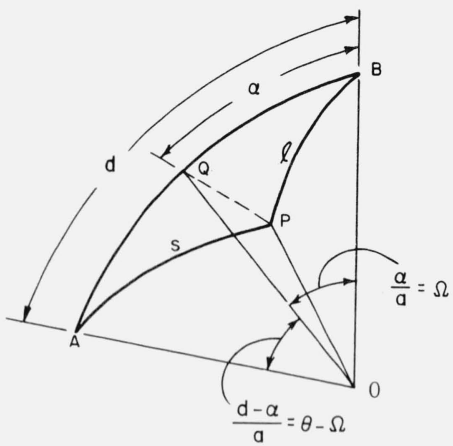

FIGURE 2. Coordinates for describing the integration over the spherical surface of the earth.

For the present development it is further assumed that $Z^{\prime}-Z$ does not vary in the transverse direction (i.e., with $\beta$ ) over the surface $S$. Thus, $Z^{\prime}$ is regarded only as a function of $\alpha$.

Within the region of validity of the present approximations, the element of area $d S$ may be approximated by $a^{2} d \Omega d \beta$. The integral now has the form

$$
\begin{aligned}
& W^{\prime}\left(Z, Z^{\prime}, d\right)=W(Z, d) \\
& \quad-\frac{i k d a}{2 \pi \eta_{0}} \int_{\alpha_{1}}^{\alpha 2} \frac{Z^{\prime}(\alpha)-Z}{\alpha(d-\alpha)} f(\alpha) W^{\prime}\left(Z, Z^{\prime}, \alpha\right) W(Z, d-\alpha) \\
& \quad \times \int_{\beta_{1}(\alpha)}^{\beta_{2}(\alpha)} \exp \left[-i(k a / 2)[\cot \Omega+\cot (\theta-\Omega)] \beta^{2}\right] d \beta d \alpha,
\end{aligned}
$$

where $\beta_{1}(\alpha)=y_{1}(\alpha) / a, \beta_{2}(\alpha)=y_{2}(\alpha) / a$. In this case, the limits of the surface $S$ are $y_{1}(\alpha) \leqslant y \leqslant y_{2}(\alpha)$ and $\alpha_{1} \leqslant \alpha \leqslant \alpha_{2}$ as indicated in figure 1. After a change of the $\beta$ variable, (17) may be written in the form

$$
\begin{array}{r}
W^{\prime}\left(Z, Z^{\prime}, d\right)=W(Z, d)-\left(\frac{i k a}{2 \pi}\right)^{1 / 2} d \int_{\alpha_{1}}^{\alpha_{2}} \frac{Z^{\prime}(\alpha)-Z}{\eta_{0}} f(\alpha) \\
\times \frac{W(Z, d-\alpha) W^{\prime}\left(Z, Z^{\prime}, \alpha\right)}{\alpha(d-\alpha)\left[\cot \left(\frac{\alpha}{a}\right)+\cot \left(\frac{d-\alpha}{a}\right)\right]^{1 / 2}} F\left(u_{1}, u_{2}\right) d \alpha
\end{array}
$$

where

$$
\begin{gathered}
F\left(u_{1}, u_{2}\right)=(i / 2)^{1 / 2} \int_{u_{1}}^{u_{2}} \exp \left(-i \pi u^{2} / 2\right) d u \\
u_{1}=(k a / \pi)^{1 / 2}[\cot \Omega+\cot (\theta-\Omega)]^{1 / 2} \beta_{1},
\end{gathered}
$$

and

$$
u_{2}=(k a / \pi)^{1 / 2}[\cot \Omega+\cot (\theta-\Omega)]^{1 / 2} \beta_{2} .
$$

As indicated before, the rapidly varying function $\exp [-i k(s+l-d)]$ in the integrand of $(9)$, determines the portions of the earth's surface which are significant. The phenomenon may be described in 
terms of Fresnel zones. These are determined by the locus of the points where

$$
k(s+l-d)=m \pi / 2(\text { for } m=1,2,3 \ldots)
$$

or, to within a good approximation,

$$
(k a / 2)[\cot (\alpha / a)+\cot ((d-\alpha) / a)] \beta^{2}=m \pi / 2 .
$$

The width of the first Fresnel zone at any point $\alpha$ is then obtained from

$$
f_{m}=2 \beta a=(2 m a \lambda)^{1 / 2}[\cot (\alpha / a)+\cot ((d-\alpha) / a)]^{-1 / 2} .
$$

The maximum width, denoted $\hat{f}_{m}$, occurs where $\alpha=d / 2$. Explicitly,

$$
\hat{f_{m}}=(m \lambda d / 2)^{1 / 2} X^{1 / 2},
$$

where

$$
X=\frac{\tan [d /(2 a)]}{d /(2 a)}=1+\frac{[d /(2 a)]^{2}}{6}+\ldots .
$$

Under most conditions $X$ may be replaced by unity even when $d$ is comparable with the earth's radius $a$. Within this approximation, the Fresnel zones are ellipses and the semiminor axes are $\hat{f}_{m} / 2$, while the semimajor axes are $(d / 2)+(m \lambda / 8)$. In the classical sense, the "first Fresnel zone" corresponds to $m=2$. It is immediately evident that $F\left(u_{1}, u_{2}\right)$ being replaced by unity in (18), is equivalent to saying that the width of the surface $S$ extends to several Fresnel zones on both sides of the propagation path.

It is convenient to rewrite (18) in the following form:

$$
\begin{aligned}
W^{\prime}\left(Z, Z^{\prime}, d\right)= & W(Z, d)-\left(\frac{i k d}{2 \pi}\right)^{1 / 2} \\
& \times \int_{\alpha_{1}}^{\alpha_{2}} \frac{Z^{\prime}(\alpha)-Z}{\eta_{0}} F\left(u_{1}, u_{2}\right) \\
& \quad \times \frac{W(Z, d-\alpha) W^{\prime}\left(Z, Z^{\prime}, \alpha\right)}{[\alpha(d-\alpha)]^{1 / 2}} d \alpha
\end{aligned}
$$

where we have made use of the trigonometric identity

$$
\left[\frac{\sin (d / a)}{\sin \left(\frac{\alpha}{a}\right) \sin \left(\frac{d-\alpha}{a}\right)}\right]^{1 / 2} \frac{1}{\left[\cot \left(\frac{\alpha}{a}\right)+\cot \left(\frac{d-\alpha}{a}\right)\right]^{1 / 2}}=1
$$

When $F\left(u_{1}, u_{2}\right)$ is set equal to one, (24) bears a formal equivalence to the one dimensional integral equation developed for groundwave propagation over mixed paths [e.g., Wait, 1964]. It is interesting to note that (24), in the form given, is not restricted to distance $d$ such that $d / a<<1$. This comes about because of the normalization factor $[(d / a) / \sin (d / a)]^{1 / 2}$ which is not included in the definition of the attenua- tion function $W(Z, d)$. In the case of groundwave propagation, this distinction is not of any consequence since $d / a$ is small in any case. However, in VLF propagation in the earth-ionospheric waveguide, the ratio $d / a$ may be comparable with unity and the normalization factor mentioned above may exceed unity by a significant amount.

Equation (24) is in a reasonably tractable form for direct numerical calculation of the attenuation function $W^{\prime}\left(Z, Z^{\prime}, d\right)$ when the limits of the surface $S$ and the surface impedance function $Z^{\prime}$ are specified. For present purposes, some simple limiting cases will be considered rather than attempting a frontal assault on (24).

\section{Two Section Path}

An important special case of the general mixed path problem is when $Z^{\prime}$ is sectionally homogeneous, as indicated in figure 3. For example, if the path between $A$ and $B$ is characterized by a constant surface impedance $Z$, from 0 to $d-d_{1}$ and $Z_{1}$ from $d-d_{1}$ to $d$, the integral equation (24) simplifies to

$$
\begin{aligned}
W^{\prime}\left(Z, Z_{1}, d\right)= & W(Z, d)-\left(\frac{i k d}{2 \pi}\right)^{1 / 2}\left(\frac{Z_{1}-Z}{\eta_{0}}\right) \\
& \times \int_{0}^{d_{1}} \frac{W(Z, d-\alpha) W^{\prime}\left(Z, Z_{1}, \alpha\right)}{[\alpha(d-\alpha)]^{1 / 2}} d \alpha,
\end{aligned}
$$

when $d_{1}>0$ and when the function $F\left(u_{1}, u_{2}\right)$ has been replaced by unity. Of course, if $d_{1}<0$ such that $A$ and $B$ are both over the surface of the earth of surface impedance $Z$, it is seen from (24) that

$$
W^{\prime}\left(Z, Z_{1}, d\right)=W(Z, d) .
$$

At least this is true to within the stationary phase approximation which, in effect, reduces the area integration to a line integration. As a result of this reasoning it is equally justified to replace $W^{\prime}\left(Z, Z_{1}, \alpha\right)$ where it occurs in the integrand of (26) with $W\left(Z_{1}, \alpha\right)$ which is the attenuation function for propagation from the point $\mathrm{B}$ to distance $\alpha$ over a homogeneous earth of surface impedance $Z_{1}$.

Equation (26) with the simplification indicated in the preceding paragraph may be written in terms of the dimensionless coordinates mentioned earlier. Thus,

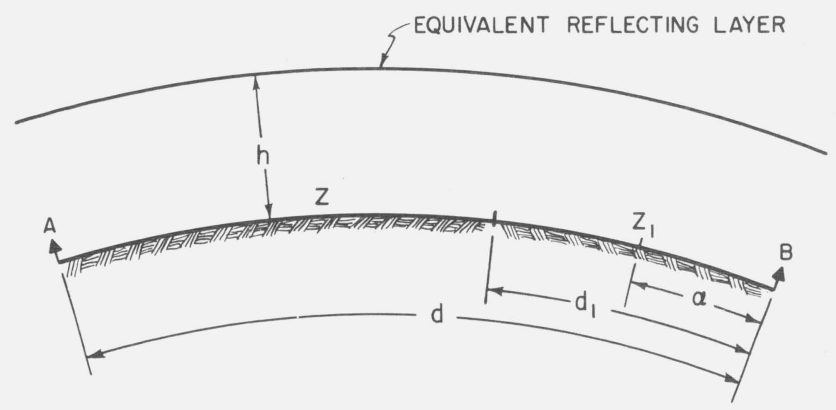

FIGURE 3. Sectional view of earth-ionosphere waveguide for a twosection path, such that $\mathrm{Z}_{1} \neq \mathrm{Z}$. 
for the two section path,

$$
\begin{aligned}
W^{\prime}\left(q, q_{1}, \chi\right) \cong W(q, \chi) & +\left(\frac{\chi}{\pi i}\right)^{1 / 2}\left(q_{1}-q\right) \\
& \times \int_{0}^{\chi_{1}} \frac{W(q, \chi-\hat{\chi}) W\left(q_{1}, \hat{\chi}\right)}{[(\chi-\hat{\chi}) \hat{\chi}]^{1 / 2}} d \hat{\chi},
\end{aligned}
$$

where

$$
\begin{aligned}
& q=-i\left(Z / \eta_{0}\right)(k a / 2)^{1 / 3}, \quad q_{1}=-i\left(Z_{1} / \eta_{0}\right)(k a / 2)^{1 / 3}, \\
& \chi=(d / a)(k a / 2)^{1 / 3}, \quad \chi_{1}=\left(d_{1} / a\right)(k a / 2)^{1 / 3}, \text { and } \\
& \hat{\chi}=(\alpha / a)(k a / 2)^{1 / 3} .
\end{aligned}
$$

Because of (4), we may write

$$
W \frac{(q, \chi-\hat{\chi})}{(\chi-\chi)^{1 / 2}}=\frac{2}{y_{0}}\left(\frac{\pi}{i}\right)^{1 / 2} \sum_{n=1,2,3 \ldots} \Lambda_{n} e^{-i(\chi-\hat{\chi}) t_{n}}
$$

and

$$
W \frac{\left(q_{1}, \hat{\chi}\right)}{(\hat{\chi})^{1 / 2}}=\frac{2}{y_{0}}\left(\frac{\pi}{i}\right)^{1 / 2} \sum_{m=1,2,3 \ldots} \bar{\Lambda}_{m} e^{-i \hat{\chi} t_{m}},
$$

where $\Lambda_{n}$ is the excitation factor corresponding to the earth of surface impedance $Z$ while $\bar{\Lambda}_{m}$ is the excitation factor corresponding to the earth of surface impedance $Z_{1}$. The $t_{n}$ are roots of (3) while $\overline{t_{m}}$ are roots of an equation identical to (3) if $q$ is replaced by $q_{1}$.

On inserting (28) and (29) into (27), the integration with respect to $\hat{\chi}$ may be readily carried out to yield

$$
\begin{aligned}
& W^{\prime}\left(q, q_{1}, \chi\right)=W(q, \chi) \\
& \quad+\frac{4}{y_{0}^{2}} e^{i 3 \pi / 4}(\pi \chi)^{1 / 2}\left(q_{1}-q\right) \sum_{n} \sum_{m} \Lambda_{n} \bar{\Lambda}_{m} e^{-i \chi t_{n}} \frac{e^{i\left(t_{n}-\bar{t}_{m}\right) \chi_{1-1}}}{\left(t_{n}-\bar{t}_{m}\right)} .
\end{aligned}
$$

The physical significance of this result is best seen by examining the special case where the distance $d-d_{1}$ or $\chi-\chi_{1}$ is sufficiently large that only the $n=1$ term is needed. Thus

$$
\begin{aligned}
W^{\prime}\left(q, q_{1}, \chi\right) \simeq & \frac{2}{y_{0}}\left(\frac{\pi}{i}\right)^{1 / 2} \Lambda_{1} e^{-i \chi \bar{t}_{1}} \\
& +\frac{4}{y_{0}^{2}} e^{i 3 \pi / 4}(\pi \chi)^{1 / 2}\left(q_{1}-q\right) \Lambda_{1} e^{-i \chi t_{1}} \\
\times & {\left[\bar{\Lambda}_{1} \frac{\left(e^{i\left(t_{1}-\bar{t}_{1}\right) \chi_{1-1}}\right)}{\left(t_{1}-\bar{t}_{1}\right)}+\bar{\Lambda}_{2} \frac{e^{i\left(t_{1}-\bar{t}_{2}\right) \chi_{1-1}}}{\left(t_{1}-\bar{t}_{2}\right)}\right.} \\
& \left.\quad+\bar{\Lambda}_{3} \frac{e^{i\left(t_{1}-\bar{t}_{3}\right) \chi_{1-1}}}{\left(t_{1}-\bar{t}_{3}\right)}+\ldots \ldots \ldots\right] .
\end{aligned}
$$

The first term on the right of (31) is the attenuation function for a path which is homogeneous throughout its length (with a surface impedance $Z$ ). The remaining terms, proportional to $q_{1}-q$ or $Z_{1}-Z$ are corrections which result from the inhomogeneity extending over the path of length $d_{1}$ (proportional to $\chi_{1}$ ). The terms proportional to $\Lambda_{2}, \Lambda_{3}$. . . etc., represent conversion of energy in the waveguide from mode 1 to mode 2,3 . . . etc.

A somewhat simpler approach to the two section problem is appropriate when the section of the path of length $d_{1}$ is very small compared with the total length of $d$ (i.e., $\chi_{1}<<\chi$ ). Then, from (27), it is seen that

$$
\begin{aligned}
W^{\prime}\left(q, q_{1}, \chi\right) \simeq W(q, \chi)+\frac{q_{1}-q}{(i \pi)^{1 / 2}} & \\
& \times \int_{0}^{\chi_{1}} W(q, \chi-\hat{\chi}) W\left(q_{1}, \hat{\chi}\right) \frac{d \hat{\chi}}{(\hat{\chi})^{1 / 2}},
\end{aligned}
$$

which is a slight simplification. When the latter result is applied to a long sea path (of length $d-d_{1}$ ) and a short land path (of length $d_{1}$ ), further simplifications are possible. For example, $W(q, \chi-\hat{\chi})$, in the integrand, is replaced by $W(q, \chi)$ and, thus,

$$
W^{\prime}\left(q, q_{1}, \chi\right)=W(q, \chi)[1+\Omega]
$$

where

$$
\Omega=\frac{q_{1}-q}{(i \pi)^{1 / 2}} \int_{0}^{x_{1}} \frac{W\left(q_{1}, \hat{\chi}\right)}{(\hat{\chi})^{1 / 2}} d \hat{\chi}
$$

On inserting the mode series expansion given by (29) into (34), the integration with respect to $\hat{\chi}$ is readily carried out. However, because of the assumed smallness of $\chi_{1}$, the resulting expansion would be very poorly convergent. An alternative approach is to recognize that $W\left(\hat{\chi}, q_{1}\right)$ may be replaced by the groundwave attenuation function for $\hat{\chi}$ ranging from 0 to $\chi_{1}$.

The form appropriate for short distances is [Wait, 1964]

$$
W\left(q_{1}, \hat{\chi}\right)=\sum_{m=0,1,2 \ldots} A_{m} e^{i m \pi / 4} q_{1}^{m}(\hat{\chi})^{m / 2},
$$

where

$$
\begin{aligned}
& A_{0}=1, A_{1}=-i \sqrt{\pi}, A_{2}=-2, A_{3}=i \sqrt{\pi}\left(1+\frac{1}{4 q_{1}^{3}}\right), \\
& A_{4}=\frac{4}{3}\left(1+\frac{1}{2 q_{1}^{3}}\right), \ldots \text { etc. }
\end{aligned}
$$

Using (35), the integration indicated in (34) is carried out to yield the following series form for the correction factor: 


$$
\begin{aligned}
\Omega & =-\frac{2 i}{\pi^{1 / 2}}\left(1-\frac{Z}{Z_{1}}\right) p_{1}^{1 / 2}\left[1-\frac{\left(\pi p_{1}\right)^{1 / 2}}{2}-\frac{2}{3} p_{1}\right. \\
& \left.+\frac{i \pi^{1 / 2} p_{1}^{3 / 2}}{4}\left(1+\frac{1}{4 q_{1}^{3}}\right)+\frac{4 p_{1}^{2}}{15}\left(1+\frac{1}{2 q_{1}^{3}}\right)+\cdots\right],
\end{aligned}
$$

where

$$
p_{1}^{1 / 2}=\left(k d_{1} / 2\right)^{1 / 2}\left(Z_{1} / \eta_{0}\right) e^{-i \pi / 4}
$$

and

$$
q_{1}=-i(k a / 2)^{1 / 3}\left(Z_{1} / \eta_{0}\right)
$$

It is seen from this series expansion that the earth curvature only influences the correction factor $\Omega$ through the higher order terms in powers of $p_{1}^{1 / 2}$. Also, if $Z$ and $Z_{1}$ correspond to sea and land respectively, the factor $1-\left(Z / Z_{1}\right)$ may be replaced by unity. Furthermore, at VLF, displacement currents in the ground are negligible, which means that $\sigma_{g_{1}}+i \epsilon_{g_{1}} \omega$ may be replaced by $\sigma_{g_{1}}$ in the definition of $p_{1}$. Thus

$$
p_{1}^{1 / 2} \simeq(1 / \lambda)\left(d_{1} / \sigma_{g_{1}}\right)^{1 / 2}(\pi / 60)^{1 / 2}
$$

is a real quantity. Then, to a first order

$$
\Omega \simeq \Omega^{\prime}+i \Omega^{\prime \prime}
$$

where

$$
\Omega^{\prime} \simeq-p_{1} \text { and } \Omega^{\prime \prime}=-\left(2 / \pi^{1 / 2}\right) p_{1}^{1 / 2} .
$$

Thus, the fractional reduction in the amplitude of the field is $p_{1}$ while the phase lag is increased by $2\left(p_{1} / \pi\right)^{1 / 2}$ radians. For example, if the length $d_{1}$ of the land path is $100 \mathrm{~km}$, for a land conductivity $\sigma_{g_{1}}$ of 1 millimho/m and a frequency of $15 \mathrm{kc} / \mathrm{s}$ (i.e., $\lambda=20 \mathrm{~km}$ ), it is easily found that

$$
\Omega^{\prime}=-2.62 \times 10^{-2} \text { and } \Omega^{\prime \prime}=-0.129 \mathrm{rad}=-7.4^{\circ} .
$$

For this example, the short land section has a negligible effect on the propagation over the total distance $d$.

\section{Propagation Ācross a Strip}

An interesting situation occurs when the path between $A$ and $B$ is homogeneous with surface impedance $Z$ everywhere except for a relatively short stretch of length $d_{2}$ where the surface impedance is a constant $Z_{2}$ as indicated in figure 4. Assuming that the inhomogeneity is effectively a strip of infinite transverse dimension, $F\left(u_{1}, u_{2}\right)$ in (24) may be replaced by unity. The resulting integral equation for the attenuation function may then be written

$$
\begin{aligned}
W^{\prime}=W & (q, \chi)+\left(\frac{\chi}{\pi i}\right)^{1 / 2}\left(q_{2}-q\right) \\
& \times \int_{\chi_{1}}^{\chi_{1}+\chi_{2}} \frac{W(q, \chi-\hat{\chi}) W^{\prime}\left(q, q_{2}, \hat{\chi}\right)}{[(\chi-\hat{\chi}) \hat{\chi}]^{1 / 2}} d \hat{\chi},
\end{aligned}
$$

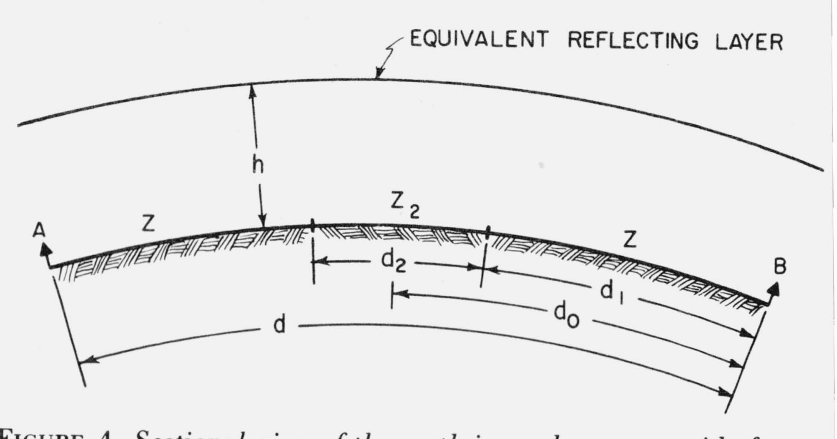

FIGURE 4. Sectional view of the earth-ionosphere waveguide for an intermediate section where $\mathrm{Z}_{2} \neq \mathrm{Z}$.

where $q_{2}=-i(k a / 2)^{1 / 3}\left(Z_{2} / \eta_{0}\right), \chi_{1}=(k a / 2)^{1 / 3} d_{1} / a$ and $\chi_{2}=(k a / 2)^{1 / 3} d_{2} / a$. The function $W^{\prime}$, as it occurs in the integrand in the preceding equation, may be identified as the attenuation function appropriate for propagation from the point $B$ to a variable point on the strip (i.e., $\chi_{1}+\chi_{2}>\hat{\chi}>\chi_{1}$ ). In accordance with the previous discussion, this particular function $W^{\prime}$ may be replaced by the appropriate form for a two section path which, in effect, ignores reflection at the boundary $\hat{\chi}=\chi_{2}$ (i.e., at distance $d_{1}+d_{2}$ from B). If the series representation given by (30) is used, the subsequent integration leads to a triply infinite series for the resultant attenuation function for the path A to B. Convergence of this type of expansion is satisfactory provided that the distance parameters $\chi, \chi_{1}$, and $\chi_{2}$ are all somewhat greater than one.

A somewhat simpler approach to the strip problem is to regard the midsection as a perturbation to the homogeneous path. In this approximation, $W^{\prime}\left(q, q_{2}, \hat{\hat{X}}\right)$ in the integrand of (38) is replaced by $W(q, \hat{\chi})$, which is the attenuation function for propagation over a homogeneous earth of surface impedance $Z$. Furthermore, if the strip is relatively narrow (i.e., $\chi_{2} \ll \chi_{1}$ and $\chi$ ), the integrand may be replaced by its value at the midpoint of the strip. Thus,

$$
\begin{aligned}
W^{\prime} \simeq W(q, \chi)+\left(\frac{\chi}{\pi i}\right)^{1 / 2}\left(q_{2}-q\right) \frac{\chi_{2}}{\left[\chi_{0}\left(\chi-\chi_{0}\right)\right]^{1 / 2}} \\
\times W\left(q, \chi-\chi_{0}\right) W\left(q, \chi_{0}\right),
\end{aligned}
$$

where

$$
\chi_{0}=\chi_{1}+\left(\chi_{2} / 2\right)=(k a / 2)^{1 / 3}\left(d_{0} / a\right), \quad d_{0}=d_{1}+\left(d_{2} / 2\right) .
$$

The perturbation term, which is proportional to $q_{2}-q$, may now be regarded as a first order or single scattering from the strip.

Using the modal representation of the form given by (28), it readily follows from (39) that

where

$$
W^{\prime} \simeq W(q, \chi)+\Delta W
$$

$$
\begin{aligned}
\Delta W=-i \frac{4 \pi}{y_{0}^{2}}\left(\frac{\chi}{\pi i}\right)^{1 / 2} & \left(q_{2}-q\right) \chi_{2} \\
& \times \sum_{n} e^{-i\left(\chi-\chi_{0}\right) t_{n}} \Lambda_{n} \sum_{m} e^{-i \chi_{0} t_{m}} \Lambda_{m} .
\end{aligned}
$$


If $\chi$ is sufficiently large, only the $n=1$ term is needed and the above representation for $\Delta W$ may be written in the more meaningful form,

$$
\begin{aligned}
\frac{\Delta W}{W} \simeq-\frac{i 2}{y_{0}} & \left(q_{2}-q\right) \chi_{2}\left[\Lambda_{1}+\Lambda_{2} e^{-i\left(t_{2}-t_{1}\right) \times 0}\right. \\
& \left.+\Lambda_{3} e^{-i\left(t_{3}-t_{1}\right) \times 0}+\Lambda_{4} e^{-i\left(t_{4}-t_{1}\right) \chi_{0}}+\ldots\right]
\end{aligned}
$$

or, what is the same thing,

$$
\begin{aligned}
\frac{\Delta W}{W} \cong-\left(\frac{Z_{2}-Z}{\eta_{0}}\right) \frac{d_{2}}{h}[ & \Lambda_{1}+\Lambda_{2} e^{-i\left(S_{2}-S_{1}\right) k d_{0}} \\
& \left.+\Lambda_{3} e^{-i\left(S_{3}-S_{1}\right) k d_{0}}+\ldots . .\right],
\end{aligned}
$$

where

$$
S_{m}=\left(1-C_{m}^{2}\right)^{1 / 2} \cong 1-\left(C_{m}^{2} / 2\right),
$$

and

$$
C_{m}=\left(-t_{m}\right)^{1 / 2}(2 / k a)^{1 / 3} \text {. }
$$

Thus, as indicated, the strip will modify the strength of the first mode by a factor proportional to $\Lambda_{1}$ and, at the same time, it will produce higher order modes proportional to $\Lambda_{2}, \Lambda_{3}, \ldots$. etc.

For negligible displacements in the earth, the multiplicative factor in (43) may be written

$-\left(\frac{Z_{2}-Z}{\eta_{0}}\right) \frac{d_{2}}{h} \simeq-\left(\frac{\epsilon_{0} \omega}{\sigma_{g_{2}}}\right)^{1 / 2}\left(1-\left(\frac{\sigma_{g_{2}}}{\sigma_{g}}\right)^{1 / 2}\right) \frac{d_{2}}{h} e^{i \pi / 4}$

where $\sigma_{g_{2}}$ is the conductivity of the earth over the strip of width $d_{2}$. For $\sigma_{g} \gg \sigma_{g_{2}}, d_{2}=100 \mathrm{~km}, h=70$ $\mathrm{km}, f=\omega / 2 \pi=15 \mathrm{kc} / \mathrm{s}, \sigma_{g_{2}}=1$ milli-mhos $/ \mathrm{m}$, it follows that

$$
\begin{aligned}
\frac{\Delta W}{W} \simeq-0.040 e^{i \pi / 4}[ & \Lambda_{1}+\Lambda_{2} e^{-i\left(S_{2}-S_{1}\right) k d_{0}} \\
& \left.+\Lambda_{3} e^{-i\left(S_{3}-S_{1}\right) k d_{0}}+. .\right] .
\end{aligned}
$$

For this example, the excitation factors $\Lambda_{1}, \Lambda_{2}, \ldots$ are not appreciably different from unity and they are nearly real. Thus the strip produces approximately a 0.03 fractional diminution of the amplitude of first mode and a change of phase of the order $0.03 \mathrm{rad}$. The higher modes are excited with a relative strength of about 0.04. The resultant effect of the higher modes, of course, depends on the magnitude of the electrical distance $k d_{0}$ and the relative phase velocities of the modes. In general, the higher modes are attenuated as a result of the increasing value of the imaginary part of $S_{m}$ as $m$ increases. As indicated above, numerical values of the excitation factors $\Lambda_{m}$ and the propagation factors $S_{m}$ are available [Wait and Spies, 1964] for a variety of conditions appropriate in the VLF range.

\section{Concluding Remarks}

It would appear that the inhomogeneity of the ground is an important factor in the propagation of VLF radio waves in the earth-ionosphere waveguide. In certain practical applications, such as to navigation systems and worldwide communications, the influence of inhomogeneous land sections on the path may alter significantly the normal behavior of the transmission. In particular, abrupt changes in conductivity from sea to land may convert appreciable amounts of energy to the higher modes. Not only will this change the resultant attenuation, but the effective phase velocity will be modified as a result of modal interference.

I thank K. P. Spies and R. L. Gallawa for their helpful comments.

\section{References}

Monteath, G. D. (1951), Application of the compensation theorem to certain radiation and propagation problems, Proc. Inst. Elec. Engrs. (London) pt. IV, 98, 23-30.

Wait, J. R. (1962), Electromagnetic Waves in Stratified Media, ch. VII (Pergamon Press Ltd., London).

Wait, J. R. (1964), Advances in Radio Research, ed. J. A. Saxton, 1, 157-217 (Academic Press, London). (Contains many references to earlier work.)

Wait, J. R. and K. P. Spies (Dec. 1964), Characteristics of the earth ionosphere waveguide for VLF radio waves, NBS Tech. Note 300. (Available from Superintendent of Documents, U.S. Government Printing Office, Wash., D.C. 20402, price 50 cents.)

\section{Additional Reference}

King, R. J., J.R. Wait, and S. W. Maley (1965), Experimental and theoretical studies of propagation of groundwaves across mixed paths, Department of Electrical Engineering, University of Colorado (to be presented at URSI Symposium on Electromagnetic Theory, Delft, Sept. 1965).

(Paper 69D7-531) 